\title{
Radiation-Induced Cervical Spinal Cord Cavernoma Following Head and Neck Radiotherapy: Case Report
}

\author{
Hosam Al-Jehani ${ }^{1,2,3}$ Ahmad Najjar ${ }^{1} \quad$ Abdulwahid Barnawi $^{1} \quad$ Daniel Shedid $^{1}$ \\ ${ }^{1}$ Department of Surgery, Hôpital Notre-Dame, Montreal, Quebec, Canada \\ 2 Department of Neurology and Neurosurgery, Montreal Neurological \\ Hospital, McGill University, Montreal, Quebec, Canada \\ ${ }^{3}$ Department of Neurosurgery, King Fahad University Hospital, Imam \\ Abdulrahman Bin Faisal University, Saudi Arabia \\ Address for correspondence Dr. Hosam M. Al-jehani, MBBS, MSc, \\ FRCSC, Department of Neurology and Neurosurgery, Montreal \\ Neurological Institute and Hospital, McGill University, 3801 University \\ Street Montreal, Room 109, Quebec H3A 2B4, Montreal, Quebec, \\ Canada (e-mail: hjehani@iau.edu.sa).
}

J Neurol Surg Rep 2020;81:e39-e41.
Abstract
Keywords
- arteriovenous malformation
- head and neck cancer
- radiation induced cavernoma
- spinal cord cavernous angiomas

Cavernous angiomas are congenital vascular malformations that affect the central nervous system. Reports implicated radiation therapy as a triggering factor for the formation of cavernomas but not in relation with head and neck radiation therapy. Radiation-induced cavernomas (RIC) should be considered in the differential diagnosis of focal neurological symptoms in any patient who has received previous cranial-spinal or head and neck radiotherapy.

\section{Introduction}

Cavernous angiomas or cavernomas belong to a subtype of central nervous system (CNS) vascular malformations characterized by thin walled, dilated capillary spaces with no intervening brain tissue. They are mostly of congenital origin with a prevalence of 0.5 to $0.6 \%$ in the general population. ${ }^{1}$

They are diagnosed as they become symptomatic either through epileptic seizures or focal neurological symptoms attributable to rupture and subsequent bleeding. The prospective hemorrhage rate has been estimated to be 0.7 to $4.2 \%{ }^{2}$ Congenital cavernomas of the spinal cord are a rare subset of CNS cavernoma and are reported to be clinically more aggressive compared with their cranial counterparts.

They can occur solely in the spinal cord or in association with cranial lesions. Patients with spinal cord cavernous malformations are at an increased risk for multiple neuraxis cavernous malformations.

De novo radiation-induced cavernomas (RIC's) have reported in response to cranial irradiation for different tumoral condition and in response to cranial as well as total body irradiation. ${ }^{3}$
The pathophysiologic mechanisms of RICs remain to be determined. Hypotheses include vulnerability of the immature brain of children receiving radiation therapy, inducing vessel wall necrosis. This will lead to endothelial swelling, dilation of the vessel lumen, hyalinization and fibrosis, which are the pathological substrate to cavernoma formation. $^{3}$

Another possibility is the radiation enhances the growth, and thus the detection of a previously small nondetectable cavernomas. The final common pathway for these hypotheses includes, but not restricted to; an increased expression of vascular endothelial growth factor (VEGF) in response to irradiation. ${ }^{3}$ RICs evolve from a multitude of interplaying factors. RICs is a phenomenon, described primarily with cranial radiotherapy and total body radiotherapy implicating the cranial compartment, in response to radiation dose of 30 Gy or more with a lag time between radiotherapy (RTX) and RICs that varies from 8 to 11.7 years. ${ }^{4}$

We report a case in which an RIC was diagnosed in the cervical spinal cord following radiotherapy to a head neck tumor. received

October 20, 2018

accepted after revision

May 20, 2019
DOI https://doi.org/

10.1055/s-0040-1709714. ISSN 2193-6358. (c) 2020 Georg Thieme Verlag KG
Stuttgart · New York

License terms

(®) (1) $\circledast$ 


\section{Case Report}

A 41-year-old man, known as a carrier for the cystic fibrosis gene, with previous medical history of asthma and lowgrade mucoepidermoid cancer of the left parotid gland diagnosed 11 years earlier, was treated with surgical resection and postoperative radiotherapy. He received 66 Gy of neutron-based radiation therapy fractionated in 33 sessions delivered to the parotid region. He presented with paresthesias of his lower limbs bilaterally together with paresthesias and weakness of right upper limb. Magnetic resonance imaging (MRI) showed a mixed signal intramedullary lesion at C3 to $\mathrm{C} 4$ region of the spinal cord consistent with cavernous angiomas (-Fig. 1). No other lesions were found in other spinal or cranial levels. He underwent a laminectomy of $\mathrm{C} 3$ to $\mathrm{C} 4$ and resection of the lesion under electrophysiological monitoring. Total resection achieved with favorable postoperative course. Postoperative, patient did well complain of mild weakness, fourfifths at shoulder right side with light pain and decrease sensation to touch and pain from C3 to T2 right side, for which the patient underwent physiotherapy. Postoperatively, he developed a paraspinal seroma, which is managed conservatively. Postoperative MRI within a week showed a collection in the soft tissue of a hematoma or seroma with light mass effect. MRI at 3 months showed complete resection of the lesion with resolution of the collection postoperatively (-Fig. 2). No new deficits were seen on postoperative evaluation and his initial paresthesia and weakness improved significantly.

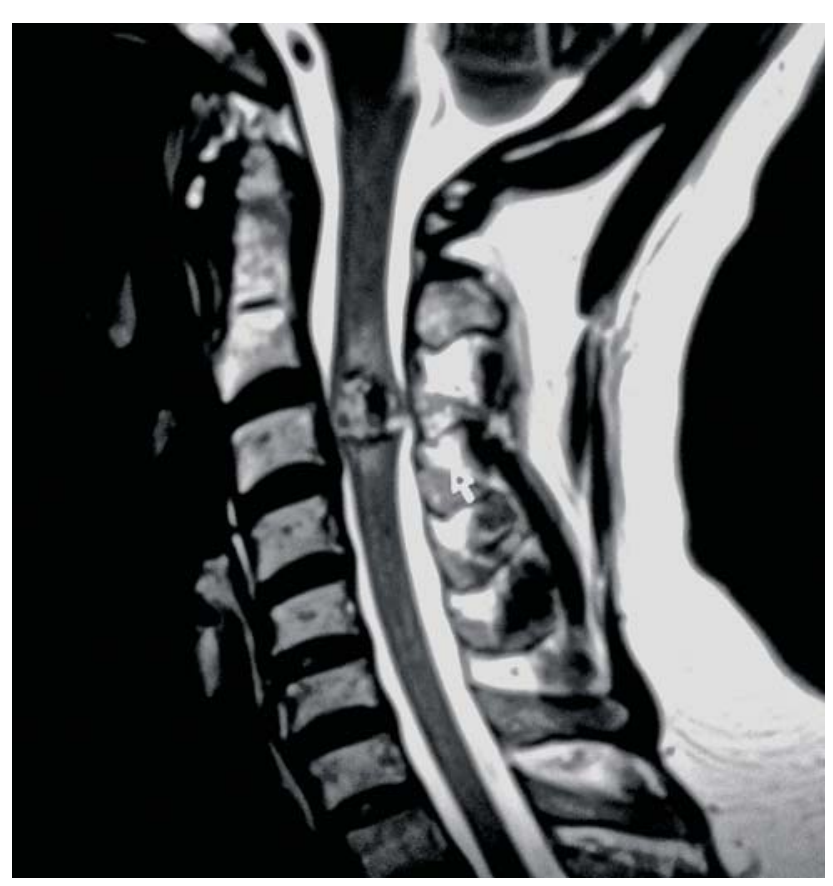

Fig. 1 Sagittal T2-weighted imaging of the cervical spine showing a spinal cavernoma at C2-C3 disc space level occupying the majority of the thickness of the spinal cord with signal change extending cephalad and caudal with no syringomyelia.

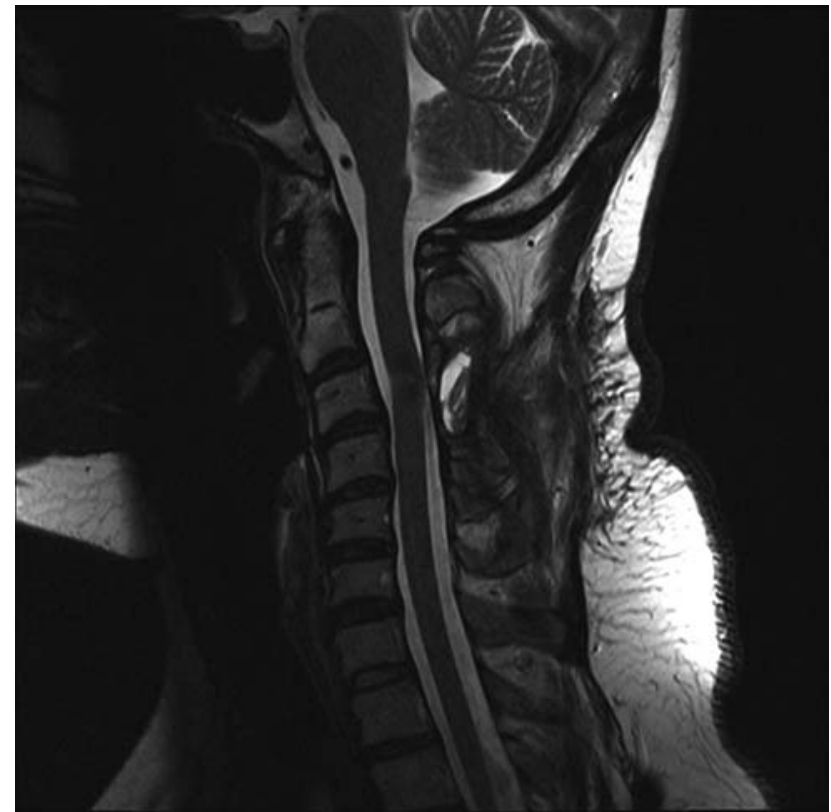

Fig. 2 Postoperative sagittal T2-weighted MRI of the cervical spine showing complete resection of the previously seen cavernoma with minimal non expansive gliotic scar with complete resolution of the previously noted cord signal change cephalad and caudal to the cavernoma. MRI, magnetic resonance imaging.

\section{Discussion}

Spinal cavernomas are rare lesions. Symptoms include sensorimotor paresis, radicular pain, and neurogenic sphincter control problems which are more common than sudden neurological deficit. Indications for microsurgical removal are progressive neurological deficit or prevention of bleeding.

The prevalence of RICs is not known. Most patients develop only a single cavernoma, whereas multiple cavernomas are extraordinary rare.

Studies linking radiation therapy to occurrence of spinal cavernoma are limited and not subject to randomization given the variability of radiation protocols given to different tumors. Of note, de novo cavernomas are thought to have an increased risk for bleeding compared with congenital cavernomas.

We report the first case of RIC post head and neck radiotherapy. Several aspects of our patient are noteworthy.

RICs are reported in the context of primary brain tumors, such as medulloblastomas, gliomas, or CNS lymphoma. ${ }^{3,4}$ In addition, a growing body of evidence indicates that hematological malignancies treated with any form of cerebral radiotherapy are prone to the risk of cerebral cavernoma development. Therefore, we present here the first adult patient with a documented history of a RIC following radiation therapy to a non-CNS, nonhematological malignancy. Furthermore, our patient is much older compared with most other reports, as RICs mainly develop in the second decade, suggesting a multifactorial role in the development of spinal RICs. ${ }^{3}$

Since our patient received a focal field of radiation, one would probably not be surprised with the development of RICs in the cervical spinal cord as it is situated along the field of radiation. 
Radiation therapy parameters implicated in the induction of RICs include increasing doses and younger age with the occurrence of RIC reported at $46 \%$ in 8 years and up to $61.5 \%$ after 25 years of radiotherapy. ${ }^{1}$ The threshold for triggering the development of RIC's is debatable, but cranial RICs have been reported with radiation doses as low as 12 to $30 \mathrm{~Gy}$.

Very few cases of RIC were reported following spinal cord field irradiation. The primary diagnoses were spinal astrocytoma, receiving focal irradiation, and infundibular germinoma, and posterior fossa medulloblastoma, both receiving craniospinal axis irradiation. The lag time between radiotherapy and the clinical presentation ranged from 5 to 13 years. All patients presented with an acute deterioration, attributable to a bleed in the cavernoma, and improved after surgical resection of the cavernomas.

Spinal cord RICs might be different. The smaller tissue target might be a reason for the less frequent development of RICs, but one might speculates that with the smaller size of neural tissue susceptible to RTX, the exposure of even a fallout radiation dose, would be sufficient to trigger chances conducive to the development of RIC of the spinal cord. It was speculated that low or lower field radiation leads to sublethal DNA damage of cells with abnormal cell growth and the formation of RICs.

These data fit well with the low-radiation dose applied to our patient as the radiation treatment was planned along the field of the parotid gland, with some radiation reaching to the cervical spinal cord.

RIC seems to carry a slightly more aggressive hemorrhage course than its congenital counterparts. Identified risk factors for hemorrhage in RIC include younger age at irradiation, prior hemorrhage, multiplicity of cavernomas, and pregnancy. ${ }^{1}$ It was also shown that infratentorial cavernomas are more likely to be associated with symptomatic bleeding compared with supratentorial caverno- mas, likely related to the compact nature of the spinal cord and it milieu. ${ }^{1}$ Flemming et al demonstrated in a cohort of incidental cavernomas that the annual rate of bleeding was $6.19 \%$ in patients initially presenting with hemorrhage, $2.18 \%$ in patients with symptoms not related to hemorrhage and $0.33 \%$ in patients with an incidental finding. ${ }^{2}$ This latter point might be a justification to screen patients receiving craniospinal irradiation for the potential development of RIC every 5 to 10 years.

RIC should be considered in the differential diagnosis of focal neurological symptoms in any patient who has received previous cranial-spinal radiotherapy. This is, especially, true for patients treated with cranial and total body radiotherapy for primary CNS tumors and hematological disorders, but should also be inclusive of head and neck radiotherapy.

Conflict of Interest

None.

\section{References}

1 Koike T, Yanagimachi N, Ishiguro $\mathrm{H}$, et al. High incidence of radiation-induced cavernous hemangioma in long-term survivors who underwent hematopoietic stem cell transplantation with radiation therapy during childhood or adolescence. Biol Blood Marrow Transplant 2012;18(07):1090-1098

2 Flemming KD, Link MJ, Christianson TJ, Brown RD Jr. Prospective hemorrhage risk of intracerebral cavernous malformations. Neurology 2012;78(09):632-636

3 Nimjee SM, Powers CJ, Bulsara KR. Review of the literature on de novo formation of cavernous malformations of the central nervous system after radiation therapy. Neurosurg Focus 2006;21 (01):e4

4 Heckl S, Aschoff A, Kunze S. Radiation-induced cavernous hemangiomas of the brain: a late effect predominantly in children. Cancer 2002;94(12):3285-3291 